\title{
Impaired explicit self-awareness but preserved behavioral regulation in patients with
}

\section{Alzheimer Disease}

Objectives: Impairments of metacognitive skills represent a critical symptom in Alzheimer Disease (AD) because it frequently results in a lack of self-awareness. However, recent findings suggest that, despite an inability to explicitly estimate their own cognitive functioning, patients might demonstrate some implicit recognition of difficulties. In this study, we tested whether a behavioral dissociation between explicit and implicit measures of metacognition can be found in both healthy older controls $(n=20)$ and AD patients $(n=20)$.

Methods: Our two groups of participants (AD vs. Controls) were asked to complete a forcedchoice perceptual identification test and to explicitly rate their confidence in each decision (i.e., explicit measure of metacognition). Moreover, they also had the opportunity to ask for a cue to help them decide if their response was correct (i.e., implicit measure of metacognition).

Results: Data revealed that all participants asked for a cue more often after an incorrect response than after a correct response in the forced-choice identification test, indicating a good ability to implicitly introspect on the results of their cognitive operations. On the contrary, only healthy participants displayed metacognitive sensitivity when making explicit confidence judgments.

Conclusion: Our findings suggest that implicit metacognition may be less affected than explicit metacognition in Alzheimer's disease.

Keywords: Alzheimer Disease; Metacognition; Monitoring; Self-awareness 


\section{Introduction}

Humans have the ability to estimate the success of their cognitive processes, a capacity that is known as metacognition (Flavell, 1979). Impairments of metacognitive skills represent a critical neuropsychiatric symptom in various clinical diagnoses such as Alzheimer's Disease (AD), frontotemporal dementia, autism, schizophrenia, or drug addiction (see Cosentino, 2014; Goldstein et al., 2009). Notably, one prominent symptom is anosognosia, or lack of selfawareness. Lack of self-awareness for behavioral or cognitive impairment is problematic for patients because it has been linked to reduced engagement in remediation strategies (Clare, Wilson, Carter, Roth, \& Hodges, 2004), poor adherence to medication (Patel \& Prince, 2001), impaired management of functional difficulties (Koltai, Welsh-Bohmer, \& Schmechel, 2001) as well as to an increase of the frequency of risky behaviors (Starkstein, Jorge, Mizrahi, Adrian, \& Robinson, 2007). Moreover, it also contributes to caregivers' burden (Seltzer, Vasterling, Yoder, \& Thompson, 1997).

Anosognosia is particularly frequent among AD patients. According to Orfei et al. (2010), for example, $42 \%$ of patients with mild $\mathrm{AD}$ could already be diagnosed as presenting a selfawareness impairment, a figure that has been shown to increase over the course of the disease (e.g., Edmonds et al., 2018). Recently, numerous studies have thus been conducted in order to better understand which specific metacognitive deficits could account for the emergence of anosognosia in AD (e.g., Chapman et al., 2018; Thomas, Lee, \& Balota, 2013). These studies have revealed that a specific impairment of metacognitive monitoring - i.e., a process by which individuals evaluate online their own functioning while performing a cognitive task - could be one of the mechanisms leading to anosognosia (Agnew \& Morris, 1998; Chapman et al., 2018; Cosentino et al., 2016). As evidence for this, Consentino et al. (2016) have found that AD patients with impaired self-awareness were unable to make accurate confidence ratings when 
completing a memory task, suggesting that these patients are not able to accurately evaluate their own memory performance.

However, despite explicit overestimation of functioning, some authors have shown that AD patients could demonstrate emotional reactivity to cognitive failure in the same extent as healthy participants, revealing some implicit recognition of difficulties. Mograbi, Brown, Salas, and Morris (2012), for instance, have conducted an experiment in which AD patients had to complete two parallel tasks: one in a success condition and one in a failure condition. Explicit metacognition was assessed by asking participants to verbally report their performance for the task (i.e. global retrospective confidence judgments). Implicit metacognition was assessed using emotional reactivity (i.e., facial expression) of the participants while performing the task. The results indicated that, relative to matched controls, AD patients exhibited impaired explicit metacognition (i.e., their explicit metacognitive judgments were not calibrated with their actual performance). However, they showed comparable emotional reactivity to failure relative to success in performing tasks. Importantly, some recent data have suggested that such implicit emotional responses to experiences of failure in cognitive tasks could result in better long-term behavioral adaptation of unaware patients (Bomilcar, Morris, Brown, \& Mograbi, 2018). Such findings are interesting because they are consistent with several theoretical frameworks which incorporate an implicit component in their model of self-awareness (e.g., the Cognitive Awareness Model - CAM, Agnew \& Morris, 1998; the Comprehensive Dynamic Interactional Model, Toglia \& Kirk, 2000). In these models, an implicit component is usually supposed to bypass explicit awareness, allowing the emergence of behavioral and affective regulation even when explicit awareness is impaired. These frameworks are also consistent with the Trace Accessibility model of metacognition proposed by Koriat (2007) which posits that metacognition is available in the form of implicit access to mental operations that can influence behavior even when it cannot be reported verbally. 
Koriat's Trace Accessibility model has recently been supported by various studies conducted in populations where explicit metacognition is not available or not fully mature as in young children (Balcomb \& Gerken, 2008; Geurten \& Bastin, 2018), infants (Goupil, RomandMonnier, \& Kouider, 2016), or non-human animals (Vining \& Marsh, 2015). For instance, Geurten and Bastin (2019) have created a procedure allowing to assess both explicit and implicit metacognition on the basis of paradigms originally developed for non-humans. They showed that this procedure can behaviorally capture young children's metacognitive skills. Specifically, their data revealed that when participants were given the possibility to ask for a cue when they were uncertain about the correctness of their response during a perceptual identification test, 2.5-year-old children had a lower rate of correct responses on items for which they required a cue than on items for which they did not require a cue, revealing an accurate implicit behavioral estimation of their performance. On the reverse, when children were explicitly asked to judge the certainty of their responses, they were unable to discriminate between their correct and incorrect identifications.

Although data from studies conducted in children and non-humans seem to support a possible dissociation between an explicit and an implicit form of metacognition, such investigations are still needed in $\mathrm{AD}$ where data remain to be gathered to determine whether and when implicit metacognition is preserved. Moreover, to our knowledge, the small number of studies which have experimentally examined the potential dissociation between an explicit and an implicit metacognition have mostly relied on paradigm exploring whether AD patients were able to process the affective valence of failure (e.g., Bomilcar et al., 2018; Mograbi et al., 2012). These experiments were not designed to test whether patients were able to behaviorally react to a poor cognitive performance, as it is postulated by the CAM model (Morris \& Hannesdottir, 2004) and the Trace Accessibility model (Koriat, 2007). 
In this context, the primary goal of this study was (a) to test whether AD patients have some metacognitive skills that could be used implicitly to regulate their performance and (b) to confirm that these skills cannot be captured by traditional explicit measures of metacognition. To this end, a paradigm inspired by the one created by Geurten and Bastin (2019) for young children has been adapted and administered to a group of AD patients and their matched controls in order to assess both their explicit and their implicit metacognitive monitoring skills. Specifically, all the participants were asked to complete a forced-choice perceptual identification test and to rate their confidence in each decision (explicit metacognitive measure). Moreover, in addition to their confidence rating, participants also had the opportunity to ask for a cue to help them decide if their response was correct (implicit metacognitive measure). Participants exhibiting explicit metacognitive abilities should demonstrate higher confidence after a correct response than after an incorrect response. Similarly, participants with implicit metacognitive abilities should be more likely to ask for a cue after inaccurate than accurate responses.

If our hypotheses are correct and that patients with $\mathrm{AD}$ are able to engage in accurate implicit metacognition, but show altered explicit metacognition as compared to matched controls, we would expect a lower accuracy for the explicit measure than for the implicit measure of metacognition. Furthermore, we would also anticipate that controls would outperform patients, but only for the explicit metacognitive index. On the reverse, if $\mathrm{AD}$ patients show an impairment for both the implicit and the explicit measure of metacognition, we would expect them to be outperformed by healthy participants for the two types of metacognitive measures. Finally, a third possible scenario could be that both AD patients and healthy participants are equally able to make accurate explicit and implicit metacognitive judgments. In this case, we would expect our two groups of participants to show a similar pattern of results for the two classes of metacognitive measures. However, given the amount of data indicating that explicit 
metacognitive monitoring processes are frequently impaired in AD (e.g., Ansell \& Bucks, 2006; Cosentino, Metcalfe, Butterfield, \& Stern, 2007; Cosentino et al., 2016; Galeone, Pappalardo, Chieffi, Iavarone, \& Carlomagno, 2011), this hypothesis appears quite unlikely, at least at the group level.

\section{Methods}

\section{Participants}

Twenty patients ( 7 females) with mild to moderate clinically probable AD and their matched controls participated at this study. Patients were recruited via collaboration with the Memory Center of the Department of Neurology of CHU Liège (Belgium). The age of the AD patients ranged from 64 to 84 years and their education level ranged from 8 to 21 years. Patients were diagnosed as having major neurocognitive disorder according to the Diagnostic and Statistical Manual of mental disorders (DSM-V) and they met clinical criteria for probable Alzheimer's Disease following the NIA-AA recommendations (McKhann et al., 2011). Their Mini Mental State Examination (MMSE) score was between 18 and 25 (Folstein, Folstein, \& McHugh, 1975). They were identified as having a relative deficit of self-awareness on the basis of an interview conducted by the neurologist who supervised their follow-up (ES) as well as on a significant discrepancy between self-reported and other-reported assessment of memory problems (i.e., a self and other-form of a questionnaire adapted from the Memory Awareness Rating Scale was used for this evaluation; Clare, Wilson, Carter, Roth, \& Hodges, 2002) as compared to healthy controls. The patients had no mental retardation, no history of psychiatric or neurological illness. They were not engaged in substance abuse and were free of medication that could negatively affect cognitive functioning.

Moreover, one healthy participant was matched with each AD patient for age, gender $(n=20$; 7 females), and education level. They were all non-institutionalized, alert, and subject to the 
same exclusion criteria as the $\mathrm{AD}$ group. Their age ranged from 63 to 85 years and their education level ranged from 7 to 20 years. The control and the patient groups did not differ significantly in age or education, all $p s>.54$. No older adults were excluded as they all had MMSE scores above $27(M=29.35)$. Patients and controls characteristics are displayed in Table 1.

$<$ Table 1 near here >

The Sample size was determined a priori to reach a predicted power of $.80(\alpha=.05, \beta=.20)$ for a double within-between interaction (small to medium effect size) with two groups and two repetitions, on the basis of a pilot study conducted on a sample of 12 patients with mild AD and their marched controls.

\section{Stimuli}

The stimuli consisted of 90 target-distractor pairs of two dimensional colored line drawings (objects and animals). During the test, target items were first presented in degraded form (40\% blurring given using the cubist filter of the GNU Image Manipulation Program). To avoid differences in our two metacognitive measures being confounded with the fact that the identification task could be easier for healthy controls than for AD patients, we controlled participants' identification performance by adjusting the level of difficulty of the targetdistractor pair of stimuli on a trial-by-trial basis. Specifically, the level of difficulty of the stimuli were adjusted using an adaptive staircase procedure (inpired by Fleming, Weil, Nagy, Dolan, \& Rees, 2010), designed to result in convergence of $65 \%$ accuracy for individual performance (i.e., above-chance performance without reaching a ceiling). This specific rate of accuracy was chosen as opposed to the more classical $70 \%$ accuracy rate in order to slightly increase the sensitivity of the metacognitive tasks and, thus, avoid potential ceiling effects at the meta-level. Indeed, previous studies have shown that providing a metacognitive judgment 
on an incorrect response was more difficult than providing a judgment on a correct response (e.g., Koriat, 2018). The level of difficulty of each pair were determined through a pretest in which 12 patients with mild $\mathrm{AD}$ and their matched controls were asked to complete four perceptual identification tests. During each test, participants were presented with 30 degraded pictures one at a time (1 s each) and then determined which of two drawings depicted the previously seen items. In all, 120 target-distractor pairs were randomly presented. Following the procedure described by Geurten and Bastin (2019), stimuli were then sorted into three categories depending on the average accuracy with which they were identified: (a) items that were correctly identified by more than two thirds of the participants in both groups (AD vs. controls) were classified as having a low level of difficulty (mean $=78.46 \%$; $\mathrm{SD}=5.2$ ); (b) items that were identified correctly by more than one third, but less than two thirds, of the participants in each group were classified as having a medium level of difficulty (mean = $51.1 \%$; SD $=7.1$ ); (c) items that were correctly identified by less than one third of the participants in each group were classified as having a high level of difficulty (mean $=19.6 \%$; $\mathrm{SD}=7.5$ ). Thirty target-distractor pairs were selected in each category to be used as stimuli in the experiment. Moreover, a set of 90 additional colored line drawings selected to be either conceptually or perceptually related to one of each target-distractor pair (e.g., Moon - Star) served as cues during the implicit judgment phase.

\section{Procedure}

This study were conducted after the approval by ethics committees of the participating institution and with written informed consent of all participants. The procedure used was the same as the one created by Geurten and Bastin (2019). Each participant underwent a session lasting approximately 20 minutes during which a perceptual identification test was completed. For each item of this test, participants went through three successive phases: (a) an identification phase, (b) an explicit judgment phase (confidence scale), and (c) an implicit 
judgment phase (cue selection). The latter two phases were counterbalanced so that half of participants in each group were presented with the explicit judgment phase first while the other half started with the implicit judgment phase (see Figure 1).

The perceptual identification test was preceded by four practice trials so that participants could become familiar with the apparatus and the general procedure of the three phases of the test. They were given feedback on the basis of the correspondence between their identification response and their cue selection or their choice on the confidence scale (e.g., if participants selected the low-confidence option after an incorrect identification, they received positive feedback). Specifically, participants received feedback on the accuracy of their explicit judgments on odd trials and on the accuracy of their implicit judgments on even trials. At the end of the practice trials, every participant was able to correctly use both the explicit and the implicit scale of confidence.

$<$ Figure 1 near here >

\section{Identification phase}

A list of 33 degraded stimuli was displayed in random order for 1 second. After each item, participants were presented with a target-distractor pair of stimuli for an unlimited time and were asked to point to the drawing they had previously seen (forced choice). The experimenter then clicked on the selected picture to validate the response. The side of the screen in which the target stimulus was displayed was randomized over the trials. After each response, a blank screen appeared for $100 \mathrm{~ms}$, followed by either the explicit or the implicit judgment phase. As previously mentioned, an adaptive staircase procedure (Fleming et al., 2010) was used to equate performance across participants. Specifically, three consecutive incorrect responses or two incorrect responses out of three trials resulted in a reduction of the level of difficulty by one step (i.e., a target-distractor pair from the lower category of difficulty was presented next), 
whereas two or more correct responses out of three trials resulted in an increase of the difficulty level by one step (i.e., a target-distractor pair from the higher category of difficulty was presented next).

\section{Explicit judgment phase}

For each trial, both groups of participants rated their confidence in their responses using a 2point pictorial scale depicting low and high confidence in the form of two arrows pointing either up (to indicate high confidence) or down (to indicate low confidence). Participants were instructed to select the high-confidence point when they were "really sure" about the correctness of their answers and to select the low-confidence point when they were "not so sure" about the correctness of their answers. The experimenter clicked on the selected picture to record the answer. No feedback was provided. Low confidence was coded as 0 and high confidence was coded as 1 . After each response, a blank screen was presented for $100 \mathrm{~ms}$ before moving to the next phase.

\section{Implicit judgment phase}

For each trial, participants had the opportunity to ask for a cue to help them to decide whether their response was correct. They were instructed to ask for a cue only when they felt they had made an error in identifying the target item. Specifically, a question (i.e., "Do you want a cue to help you to decide whether your response is correct?") and two response buttons appeared in the center of the screen. Participants had to say aloud whether they wanted a cue. The experimenter pressed the "yes" button when a cue was requested and pressed the "no" button otherwise. Each choice was followed by a blank screen for $100 \mathrm{~ms}$. No feedback was provided. Accepting the cue was coded 0 and declining the cue was coded 1 . Depending on the experimental order, the cue appeared either directly after the blank screen or after the explicit judgment phase. In that way, participants' explicit judgment was never affected by the 
presentation of the cue. The cue, a picture either perceptually or conceptually related to the correct answer, appeared in the center of the screen for 3 seconds, followed by a blank screen for $100 \mathrm{~ms}$. After completing these three phases, participants were once again presented with the target-distractor pairs and asked whether they would like to change their previous answer, then they moved on to the next trial.

\section{Results}

Differences were considered as significant when the $p$ value was $<.05$. Preliminary analyses indicated no gender or phase order effects on any of the dependent variables, $t s<1.56$; $p s>.12$; further analyses were thus run on data collapsed across gender and phase order.

\section{Correct response rate}

First, to confirm that the identification performance were roughly equal between the $A D$ patients and healthy older adults, we conducted a $t$ test to compare the accuracy rate of these two groups. Performance accuracy appeared well regulated by the staircase procedure, as performance reached the predicted level and no significant differences were found between the $\operatorname{AD}(M=.67, S D=.10$, range $.59-.77)$ and the control group $(M=.68, S D=.10$, range $.57-.78)$, $t(38)=.36, p=.72, d=.14$.

\section{Implicit vs. explicit judgment}

To show a dissociation between the explicit and implicit measures of metacognition, we directly compared the accuracy of participants' confidence ratings with the accuracy of their cue selection. To do so, we calculated a measure of metacognitive sensitivity for both metacognitive measures: the A'ROC index (Galvin, Podd, Drga, \& Whitmore, 2003). A'ROC is a nonparametric and bias-free measure (i.e., theoretically uninfluenced by the overall propensity of a participant to give high or low judgments) from signal detection theory that plots the concordances (e.g., a high-confidence judgment on correct identification) against the 
discordances (e.g., a high-confidence judgment on incorrect identification). An A'ROC of 0.5 indicates no metacognitive discrimination between correct and incorrect responses. However, despite being bias-free, A'ROC could still be affected by variations in the performance at the cognitive level. One approach to deal with this problem is to use techniques to control for interindividual differences in performance through, for example, the implementation of a staircase procedure (see Fleming et al., 2014).

We first examined whether both groups of participants were able to make above-chance explicit and implicit judgments. The results of the $t$ tests showed that the metacognitive coefficients were significantly above chance in both groups for the implicit measure, $t s>5.22$, $p s<.001$, $d s>1.2$. However, if healthy older adults also showed above chance sensitivity for the explicit measure of metacognition, $t(38)=5.63, p<.001, d=1.26$, no significant differences against 0.5 were found in the $\mathrm{AD}$ patients, $t(38)=0.64, p=.53, d=.15$.

To determine whether there were a significant difference between these two judgments when they were compared directly, we then conducted a 2 (Group: AD vs. Control) x 2 (Measure: explicit vs. implicit) mixed ANOVA on the A'ROC index. The results are presented in Figure 2. They indicated that participants' metacognitive accuracy was lower for the explicit measure $(M=.56, S D=.13)$ than for the implicit measure $(M=.62, S D=.10), F(1,38)=6.75, M S e=$ $0.06, p=.01, \eta^{2}=.07$. This result is qualified by the significant group $\mathrm{x}$ measure interaction, $F(1,38)=6.51, M S e=0.06, p=.01, \eta^{2}=.08$. This interaction revealed that AD patients $(M=$ $.52 ; S D=16)$ had lower metacognitive accuracy than older adults $(M=.61 ; S D=.08)$ for the explicit measure of metacognition, $F(1,38)=4.33, M S e=0.07, p=.044, \eta^{2}=.12$, while this difference was not found for the implicit measure, $F(1,38)=0.64, M S e=0.01, p=.43, \eta^{2}=.00$ $(M=.62, S D=.10$ vs. $M=.61, S D=.09$, for the AD group and the control group, respectively). No other effect was found, $F=0.97, p=.33$. 
Finally, in a more exploratory way, correlation analyses were conducted to determine whether explicit and implicit measures of metacognition were related to participants' general cognitive functioning (i.e., MMSE scores). Results revealed a significant correlation with the explicit measure of metacognition in both $\mathrm{AD}$ patients, $r=.54, p=.01$, and control participants, $r=$ $.43, p=.04$. However, no correlations were found with the implicit metacognitive measure, all $r s<.10 p s>.20$.

\section{Discussion}

The primary goal of the present experiment was to document the existence and preservation of some implicit metacognitive monitoring skills dissociated from explicit metacognitive processes in patients with mild to moderate $\mathrm{AD}$ who were clinically considered to present selfawareness deficits. For this purpose, we adapted and used an experimental paradigm allowing participants to ask for a cue when they felt uncertain about their response and we compared this implicit measure of metacognition to a more classical explicit metacognitive measure. Moreover, in this study, we also took care to control for individual performance at the cognitive level in order to ensure that differences observed at the metacognitive level between our two groups were not due to changes in task performance (Fleming et al., 2010).

Consistent with the hypothesis that $\mathrm{AD}$ patients have some preserved form of metacognitive skills, our results reveal that both AD patients and healthy controls asked for a cue more often after an incorrect response than after a correct response in the forced-choice identification test, as revealed by a metacognitive index of accuracy higher than chance in both groups. This pattern indicates a good ability of both healthy and AD participants to implicitly estimate their own cognitive performance. Regarding the explicit measure of metacognition, however, control participants - but not AD patients - displayed a metacognitive sensitivity that differs from chance when making explicit confidence judgments. Taken together, these findings suggest a 
preservation of implicit metacognition in $\mathrm{AD}$ patients that is dissociated from their impaired explicit metacognition.

From a theoretical perspective, these results are consistent with the CAM model which posits that an implicit retrieval mechanism allows participants to regulate their cognitive functioning at both a behavioral and an affective level (Morris \& Hannesdottir, 2004). Importantly, the present study is among the first to provide direct evidence of an implicit behavioral regulation in $\mathrm{AD}$ patients. Indeed, previous experiments exploring the implicit component of the CAM model have mostly relied on procedure assessing AD patients' affective responses to cognitive failures (e.g., Mograbi et al., 2012). To date, only clinical observation were available to support the hypothesis that $\mathrm{AD}$ patients may demonstrate some signs of behavioral reactions to cognitive errors, even when they are not able to explicitly report these errors (Mograbi \& Morris, 2013). Furthermore, our findings also support the Trace Accessibility model (Koriat, 2007) which proposes that some aspects of metacognitive operations are computed automatically, without explicit access to the results of this evaluation. Within this framework, our data are interesting because, if previous studies have found that implicit metacognition could emergence very early in the course of development (e.g., Balcomb \& Gerken, 2008; Geurten \& Bastin, 2018), our findings reveal that implicit metacognition could also be more resistant than explicit metacognition to cerebral degeneration effects, at least in the case of Alzheimer's disease.

These results, if there are confirmed, could have several major practical applications. Indeed, the data collected in this study provide critical information about the nature of possibly preserved metacognitive abilities in AD. This knowledge could be crucial for future research aiming at implementing revalidation programs based on patients preserved skills in order to help them to circumvent the negative impact of poor self-awareness in various aspects of their life. This could be particularly important if, as postulated by Bomilcar et al. (2018), further 
examinations revealed that spared implicit metacognition is associated with better functional regulation in $\mathrm{AD}$ patients.

However, the preservation of implicit metacognition does not appear to be universal in $\mathrm{AD}$, as suggested by heterogeneity within the sample. Indeed, in the present experiment, only $45 \%$ of our patients $(n=9)$ presented impaired explicit metacognition (i.e., at chance metacognitive accuracy index) accompanied by preserved implicit metacognition (i.e., above chance metacognitive accuracy index). Among the remaining 55\%, 25\% $(\mathrm{n}=5)$ presented both spared implicit and explicit processes - although the accuracy of their implicit judgments remained usually higher than the accuracy of their explicit judgments,$- 15 \%(\mathrm{n}=3)$ presented an alteration of both types of metacognitive processes, and $15 \%(\mathrm{n}=3)$ demonstrated impaired implicit metacognition, but preserved explicit metacognition. These findings are, of course, to be further examined using procedure specifically designed to explore performance dissociation at the individual level. One factor that could account for this heterogeneity is the possible presence of visual agnosia in some of our AD patients. Indeed, our patients were not screened for visual agnosia before being included in the sample. Although the staircase procedure used here prevented individual differences at the cognitive level to influence the metacognitive performance, this hypothesis certainly deserved to be further investigated. However, in addition to provide preliminary evidence in favor of a possible double dissociation between explicit and implicit metacognitive monitoring processes, the pattern of results observed in this study is crucial because it indicates that the lack of self-awareness of some AD patients may result from an impairment of implicit metacognition rather than from an alteration of explicit metacognitive processes. Although there is still much to be done to understand the effect of such an implicit deficit on patients' functioning, this line of research could prove crucial to understand selfawareness deficits in AD. 
In this context, future studies should be conducted to determine the nature of the variables that are involved in the apparition of a decrease in implicit metacognitive accuracy (i.e., in contrast to explicit metacognition, implicit metacognition was not found to be related to general cognitive functioning). Future research could also be conducted to determine whether a good implicit behavioral regulation always comes with a good implicit affective regulation, as postulated by the CAM model, or if a dissociation can be found between these two processes. Furthermore, in a clinical perspective, experiments should also be carried out to explore the functional impact of impaired implicit metacognition. For instance, studies should be conducted to examine whether patients with preserved implicit metacognition better manage their functional difficulties or show lower exposure to risky behaviors than patients with reduced implicit metacognitive skills. Such findings would contribute to improve our understanding of the heterogeneity of anosognosia in $\mathrm{AD}$ and would provide new leads to help us dealing with the many issues raised by unawareness in patients' daily life. 


\section{References}

Agnew, S. K., \& Morris, R. G. (1998). The heterogeneity of anosognosia for memory impairment in Alzheimer's disease: A review of the literature and a proposed model. Aging \& Mental Health, 2, 7-19. doi:10.1080/13607869856876

Ansell, E. L., \& Bucks, R. S. (2006). Mnemonic anosognosia in Alzheimer's disease: A test of Agnew and Morris (1998). Neuropsychologia, 44, 1095-1102. doi:10.1016/j.neuropsychologia.2005.10.019

Balcomb, F. K., \& Gerken, L. (2008). Three-year-old children can access their own memory to guide responses on a visual matching task. Developmental Science, 11, 750-760. doi:10.1111/j.1467-7687.2008.00725.x

Bomilcar, I., Morris, R. G., Brown, R. G., \& Mograbi, D. C. (2018). Implicit Behavioral Change in Response to Cognitive Tasks in Alzheimer Disease. Cognitive and Behavioral Neurology, 31, 2-12. doi:10.1097/wnn.0000000000000145

Chapman, S., Colvin, L. E., Vuorre, M., Cocchini, G., Metcalfe, J., Huey, E. D., \& Cosentino, S. (2018). Cross domain self-monitoring in anosognosia for memory loss in Alzheimer's disease. Cortex, 101, 221-233. doi: 10.1016/j.cortex.2018.01.019

Clare, L., Wilson, B. A., Carter, G., Roth, I., \& Hodges, J. R. (2002). Assessing awareness in early-stage Alzheimer's disease: Development and piloting of the Memory Awareness Rating Scale. Neuropsychological Rehabilitation, 12, 341-362. doi:10.1080/09602010244000129

Clare, L., Wilson, B. A., Carter, G., Roth, I., \& Hodges, J. R. (2004). Awareness in Early-Stage Alzheimer's Disease: Relationship to Outcome of Cognitive Rehabilitation. Journal of Clinical and Experimental Neuropsychology, 26, 215-226. doi:10.1076/jcen.26.2.215.28088 
Cosentino, S. (2014). Metacognition in Alzheimer's disease The cognitive neuroscience of metacognition. (pp. 389-407). New York, NY, US: Springer-Verlag Publishing.

Cosentino, S., Metcalfe, J., Butterfield, B., \& Stern, Y. (2007). Objective Metamemory Testing Captures Awareness of Deficit in Alzheimer's Disease. Cortex, 43, 1004-1019. doi:10.1016/S0010-9452(08)70697-X

Cosentino, S., Zhu, C., Bertrand, E., Metcalfe, J., Janicki, S., \& Cines, S. (2016). Examination of the metacognitive errors that contribute to anosognosia in Alzheimer's disease. Cortex, 84, 101-110. doi:10.1016/j.cortex.2016.08.003

Edmonds, E. C., Weigand, A. J., Thomas, K. R., Eppig, J., Delano-Wood, L., Galasko, D. R., . . Bondi, M. W. (2018). Increasing Inaccuracy of Self-Reported Subjective Cognitive Complaints Over 24 Months in Empirically Derived Subtypes of Mild Cognitive Impairment. Journal of the International Neuropsychological Society, 24, 842-853. doi:10.1017/S1355617718000486

Flavell, J. H. (1979). Metacognition and cognitive monitoring: A new area of cognitivedevelopmental inquiry. American Psychologist, 34, 906-911. doi:10.1037/0003066X.34.10.906

Fleming, S. M., Weil, R. S., Nagy, Z., Dolan, R. J., \& Rees, G. (2010). Relating introspective accuracy to individual differences in brain structure. Science, 329, 1541-1543. doi:10.1126/science. 1191883

Folstein, M. F., Folstein, S. E., \& McHugh, P. R. (1975). "Mini-mental state": a practical method for grading the cognitive state of patients for the clinician. Journal of psychiatric research, 12, 189-198.

Galeone, F., Pappalardo, S., Chieffi, S., Iavarone, A., \& Carlomagno, S. (2011). Anosognosia for memory deficit in amnestic mild cognitive impairment and Alzheimer's disease. International Journal of Geriatric Psychiatry, 26, 695-701. doi:10.1002/gps.2583 
Galvin, S. J., Podd, J. V., Drga, V., \& Whitmore, J. (2003). Type 2 tasks in the theory of signal detectability: Discrimination between correct and incorrect decisions. Psychonomic Bulletin \& Review, 10, 843-876. doi:10.3758/bf03196546

Geurten, M., \& Bastin, C. (2019). Behaviors speak louder than explicit reports: Implicit metacognition in 2.5-year-old children. Developmental Science, 22, e12742. doi:10.1111/desc. 12742

Goldstein, R. Z., Craig, A. D., Bechara, A., Garavan, H., Childress, A. R., Paulus, M. P., \& Volkow, N. D. (2009). The Neurocircuitry of Impaired Insight in Drug Addiction. Trends in Cognitive Sciences, 13, 372-380. doi:https://doi.org/10.1016/j.tics.2009.06.004

Goupil, L., Romand-Monnier, M., \& Kouider, S. (2016). Infants ask for help when they know they don't know. Proceedings of the National Academy of Sciences, 113, 3492-3496. doi:10.1073/pnas.1515129113

Koltai, D. C., Welsh-Bohmer, K. A., \& Schmechel, D. E. (2001). Influence of anosognosia on treatment outcome among dementia patients. Neuropsychological Rehabilitation, 11, 455-475. doi:10.1080/09602010042000097

Koriat, A. (2007). Metacognition and consciousness. In P. D. Zelazo, M. Moscovitch, \& E. Thompson (Eds.), The Cambridge handbook of consciousness (pp. 289-325). Cambridge, UK: Cambridge University Press.

Koriat, A. (2018). When reality is out of focus: Can people tell whether their beliefs and judgments are correct or wrong? Journal of Experimental Psychology: General, 147(5), 613-631. doi:10.1037/xge0000397

McKhann, G. M., Knopman, D. S., Chertkow, H., Hyman, B. T., Jack, C. R., Kawas, C. H., . . - Phelps, C. H. (2011). The diagnosis of dementia due to Alzheimer's disease: Recommendations from the National Institute on Aging-Alzheimer's Association 
workgroups on diagnostic guidelines for Alzheimer's disease. Alzheimer's \& Dementia, 7, 263-269. doi:10.1016/j.jalz.2011.03.005

Mograbi, D. C., Brown, R. G., Salas, C., \& Morris, R. G. (2012). Emotional reactivity and awareness of task performance in Alzheimer's disease. Neuropsychologia, 50, 20752084. doi:10.1016/j.neuropsychologia.2012.05.008

Mograbi, D. C., \& Morris, R. G. (2013). Implicit awareness in anosognosia: Clinical observations, experimental evidence, and theoretical implications. Cognitive Neuroscience, 4, 181-197. doi:10.1080/17588928.2013.833899

Morris, R. G., \& Hannesdottir, K. (2004). Loss of 'awareness' in Alzheimer's disease. Cognitive neuropsychology of Alzheimer's disease, 275-296.

Orfei, M. D., Blundo, C., Celia, E., Casini, A. R., Caltagirone, C., Spalletta, G., \& Varsi, A. E. (2010). Anosognosia in Mild Cognitive Impairment and Mild Alzheimer's Disease: Frequency and Neuropsychological Correlates. The American Journal of Geriatric Psychiatry, 18, 1133-1140. doi:10.1097/JGP.0b013e3181dd1c50

Patel, V., \& Prince, M. (2001). Ageing and mental health in a developing country: who cares? Qualitative studies from Goa, India. Psychological Medicine, 31, 29-38.

Seltzer, B., Vasterling, J. J., Yoder, J., \& Thompson, K. A. (1997). Awareness of Deficit in Alzheimer's Disease: Relation to Caregiver Burden1. The Gerontologist, 37, 20-24. doi:10.1093/geront/37.1.20

Starkstein, S. E., Jorge, R., Mizrahi, R., Adrian, J., \& Robinson, R. G. (2007). Insight and danger in Alzheimer's disease. European Journal of Neurology, 14, 455-460. doi:10.1111/j.1468-1331.2007.01745.x

Thomas, A. K., Lee, M., \& Balota, D. A. (2013). Metacognitive monitoring and dementia: How intrinsic and extrinsic cues influence judgments of learning in people with early-stage Alzheimer's disease. Neuropsychology, 27, 452-463. doi:10.1037/a0033050 
Toglia, J., \& Kirk, U. (2000). Understanding awareness deficits following brain injury. NeuroRehabilitation, 15, 57-70.

Vining, A. Q., \& Marsh, H. L. (2015). Information seeking in capuchins (Cebus apella): A rudimentary form of metacognition? Animal Cognition, 18, 667-681. doi:10.1007/s10071-015-0835-7 
Table 1.

Demographic Data and Cognitive Performance for AD patients and their matched controls.

\begin{tabular}{lcc}
\hline & Healthy Controls & AD \\
\hline Age (years) & $73.5(5.1)$ & $74.6(5.8)$ \\
Education (Years) & $12.2(4.1)$ & $13.0(3.6)$ \\
Sex (male : female) & $13: 7$ & $13: 7$ \\
MMSE & $29.3(0.8)$ & $21.2(4.3)^{*}$ \\
Hit rate for perceptual task & $0.67(0.13)$ & $0.68(0.12)$
\end{tabular}

Note. $\mathrm{AD}=$ Alzheimer Disease $; \mathrm{MMSE}=$ Mini Mental State Examination; Hit rate $=$ rate of correct identification for the perceptual identification task. * Significant differences between groups with a $\mathrm{p}<.001$ 


\section{Figure Captions}

Figure 1. Description of the experimental procedure.

Figure 2. Metacognitive accuracy for the implicit (cue) and explicit (RCJ) measure of metacognitive monitoring in both $\mathrm{AD}$ patients and their matched controls; $\mathrm{RCJ}=$ Retrospective Confidence Judgment. 